from CNTW R\&D department. We briefly consulted with staff regarding themes relevant to temporary nurse workers. They expressed concern that staff perceived to be "other" would be at more risk.

Employees' age, ethnicity, employment status, nationality, length of service and seniority are routinely collected for the running of the trust. Therefore, these were anonymously collated then cross-referenced with violence and aggression incident reports (VA IR1s). Chi-squared was used to identify statistical significance. Ethno-national status was taken from self-report. We could not control for hours worked nor could we get agency staff demographic data.

We compared "exposure to at least one violent incident" in June, July and August 2019 against the following demographic categories:

Substantive vs bank staff

Band 5 and above vs band 4 and below

Staff with $<1$ year of service vs staff with $\geq 1$ year of service

"White British" staff vs Non-"White British" staff

"British" staff on self-report vs "Non-British" staff

Age $\leq 30$ years vs $\geq 31$ years

A minimum of 1682 nursing staff were analysed for each category in each month.

Result. Substantive staff, "White British", "British", younger, and staff of shorter employment length had greater frequencies of at least one VA IR1s compared to the complementary groups. Length of service was significant only in two months but judged significant overall. There was no statistically significant correlation with seniority. Substantive staff have three times the risk vs bank staff, perhaps mediated by hours worked. Other risk ratios were in the region $\mathrm{x} 1.2$ to $\mathrm{x} 1.8$.

Conclusion. Being British, White British, younger, less experienced or substantive staff correlate with subjection to reported aggression. This did not fit with staff speculation during consultation. Survival effects may be relevant. We are working to get more detailed information. Induction may help reduce aggression against newer staff.

The improvement of the quality of medical reviews of patients in seclusion in Rampton Hospital

Emma McPhail* and Ian Yanson

Rampton Hospital

${ }^{*}$ Corresponding author.

doi: 10.1192/bjo.2021.874

Aims. Improve and standardise the quality of medical seclusion reviews (MSRs).

Acknowledge existing good practise.

Highlight areas for improvement.

Improve the awareness of doctors performing MSRs of the requirements in the Mental Health Act Code of Practice (MHA CoP)

Background. MSRs are an essential clinical tool to ensure safe and consistent patient care. Patients detained in seclusion can be at heightened risk of poor mental and physical health, in addition to being a risk to themselves and others. There is clear guidance in the MHA CoP regarding what areas require to be covered in a MSR. Method. A retrospective audit of all MSRs in September 2019 across all patients within all directorates within Rampton Hospital was undertaken. 281 inpatients were identified within Rampton Hospital, and 61 of these patients were found to have had seclusion in September 2019. A total of 439 MSRs were identified for these patients.
The standard applied was the MHA CoP guidance for MSRs:

1) MSRs should be conducted in person, and should include:

2) Review of physical health

3) Review of psychiatric health

4) Assessment of the adverse effects of medication

5) Review of observations required

6) Reassessment of medication prescribed

7) Assessment of the patient's risk to others

8) Assessment of the patient's risk of self-harm

9) Assessment of the need for continuing seclusion

$100 \%$ compliance with targets or a reason why it was not possible was expected to be documented.

Result. The results show there is a large variation in compliance with the MHA CoP. The area with the highest compliance was the completion of reviews in person-(99.3\%). The criterion with the average worst compliance was whether the need for physical observations was reviewed-(4.3\%). Physical health was reviewed in $86.1 \%$ of cases, in contrast to psychiatric health at $38.3 \%$. The adverse effects of medication and reassessment of medication prescribed were recorded in only $8.9 \%$. The risk from the patient to others was recorded in $25.3 \%$, whereas risk to self was recorded in $10.7 \%$. The need for continuing seclusion was recorded in $72.7 \%$.

Conclusion. The quality of MSRs at Rampton Hospital is currently inadequate. Improvement in practice is required to meet accepted standards and ensure safe, consistent patient care. Ways to improve this are being considered, including improving the knowledge of the MHA CoP and providing a MSR template.

\section{Compliance with nice guidelines for management of depression in a community mental health team}

Sophie Mellor ${ }^{1 \star}$, Shay-Anne Pantall ${ }^{2}$ and Lisa Brownell ${ }^{2}$

${ }^{1}$ University of Birmingham, Medical School and ${ }^{2}$ Birmingham and Solihull Mental Health NHS Foundation Trust

${ }^{\star}$ Corresponding author.

doi: 10.1192/bjo.2021.875

Aims. To evaluate compliance within a Community Mental Health Team (CMHT) to the NICE guidelines for the management of depression.

Background. Reducing the prevalence of depression continues to be a major public health challenge.

Given the complexity and recurrent nature of the condition, the NICE guideline CG90 is an invaluable resource to aid the effective management of depression. Here we present an audit of adherence to this guideline within a CMHT.

Method. A retrospective electronic casenote review of all patients diagnosed with depression between January 2016 and October 2019 under the care of a Birmingham CMHT $(n=35)$, assessing key performance areas including: quality of assessment and coordinated care, risk assessment, choice of pharmacological and psychological treatment using the stepped care model and appropriate crisis resolution planning.

Result. Key results include:

The majority of patients were Caucasian (63\%). Ages ranged from 27 to 69 (mean age 48 years old).

Severity of disorder was typically moderate $(46 \%)$ or severe $(48 \%)$. Of those with a diagnosis of severe depression, $41 \%$ had associated psychotic symptoms. 
Psychiatric comorbidity was high (49\%), of which generalised anxiety disorder was the most common (59\%).

Referrals were typically from primary care $(77 \%)$. Approximately half $(51 \%)$ had reported suicidal thoughts according to the referral.

A quarter of patients $(26 \%)$ were seen by CMHT within 8 weeks of referral; $20 \%$ of referrals however waited over 12 months before being assessed.

Risk assessments were out of date for $71 \%$ of patients.

$100 \%$ of patients had a crisis plan noted within their most recent clinic letter; however, none of these met the required standards.

Polypharmacy was common (60\%), with $34 \%$ prescribed two antidepressants. Use of lithium augmentation was uncommon, with only one patient prescribed this. $43 \%$ were prescribed an antipsychotic; of which, $29 \%$ had appropriate physical health monitoring completed.

Over half of patients (60\%) had been referred to psychology services; of these, $38 \%$ had either completed or were in ongoing treatment at the time of review.

Conclusion. CMHTs manage the care of individuals with depression who have high levels of active symptoms and disability, psychiatric comorbidity, care requirements, and complex treatment plans. Pharmacological management was broadly in line with guidelines, and rates of referral to psychology were satisfactory. Risk assessment and crisis planning are clear areas in need of urgent attention in order to comply with guidelines and ensure patient safety.

\section{Epidemiology and current treatment patterns of treatment-resistant depression in Scotland: a CPRD study}

Timothy Ming ${ }^{2 \star}$, Tom Denee ${ }^{1}$, Gemma Scott ${ }^{1}$, Joachim Morrens ${ }^{2}$ and Christopher Weatherburn ${ }^{3}$

${ }^{1}$ Janssen-Cilag Ltd; ${ }^{2}$ Janssen Cilag and ${ }^{3}$ Dundee Health and Social Care Partnership

${ }^{\star}$ Corresponding author.

doi: 10.1192/bjo.2021.876

Aims. To assess the incidence and treatments currently used in clinical practice for the treatment of treatment-resistant depression (TRD) in Scotland.

Background. Patients with major depressive disorder (MDD) who have not responded to at least two successive antidepressant (AD) treatments in a single episode are described as having TreatmentResistant Depression (TRD). Epidemiological data on TRD in Scotland is lacking. Furthermore, there is no data to our knowledge on therapies prescribed in Scottish clinical practice to treat TRD.

Method. A retrospective, longitudinal cohort study was conducted using Clinical Practice Research Datalink (CPRD) medical records. Adult patients were indexed on $\mathrm{AD}$ prescription, requiring MDD diagnosis within 90 days, from Jan 2011-May 2018 with 360-day baseline and 180-day minimum follow-up periods. Failure of $\geq 2$ adequate oral AD regimens following indexing constituted TRD classification. Incidence rates of MDD and TRD (within the MDD cohort) and treatment lines following TRD classification were derived.

Result. The analysis included 20,059 patients with MDD (mean age 44 years, $63 \%$ female, median follow-up 59 months); 1,374 (6.8\%) were classified as TRD. Median time-to-TRD classification was 25 months. The incidence rate of MDD was 15.9 per 1,000 patient-years and for TRD was 14.7 per 1,000 MDD-patientyears. For all first four post-TRD treatment lines, SSRI monotherapy was the most commonly prescribed therapy, followed by combination (dual/triple) therapy and augmentation therapy (at least one oral $\mathrm{AD}$ supplemented with lithium, an antipsychotic or an anticonvulsant therapy). At first-line of TRD treatment, $1,050(76.4 \%)$ patients received monotherapy AD, 212 (15.4\%) received combination $\mathrm{AD}$ therapy and $112(8.2 \%)$ received augmentation therapy. The most common monotherapy treatments at first-line TRD were sertraline (15.6\%), mirtazapine (13.8\%), fluoxetine (12.2\%) and venlafaxine (11.6\%). Among combination therapies, mirtazapine, venlafaxine, sertraline and amitriptyline were frequently used. Among the TRD and MDD cohort, no somatic treatments were coded in CPRD, although the use of these treatments was likely underestimated.

Conclusion. Monotherapy AD treatment was the most common therapy type for all four post-TRD treatment lines. These data support the need for new treatments that can achieve and maintain therapeutic response, and avoid continuous cycling through similar AD therapies.

This study was sponsored by Janssen Cilag Ltd.

\section{COVID-19 and young people with intellectual} disability: a service review

Omer Minhas ${ }^{1 \star}$, Pippa Mundy ${ }^{2}$ and Jessica Stewart ${ }^{2}$

${ }^{1}$ Cwm Taf Morgannwg University Health Board and ${ }^{2}$ Cardiff and

Vale University Health Board

${ }^{*}$ Corresponding author.

doi: 10.1192/bjo.2021.877

Aims. A service review of specialist child and adolescent intellectual disability provision in South Wales was conducted between March and May 2020. The purpose was to explore the impact of the first COVID-19 pandemic lockdown on children with intellectual disability and their families. The review aimed to explore if the disruption to the systems involved in their care would impact their wellbeing and behavioural presentations. We also measured if there had been an increase in the use of medication. Our focus was on the distress calls, which are requests for urgent clinical review to prevent a crisis.A service review of specialist child and adolescent intellectual disability provision in South Wales was conducted between March and May 2020. The purpose was to explore the impact of the first COVID-19 pandemic lockdown on children with intellectual disability and their families. The review aimed to explore if the disruption to the systems involved in their care would impact their wellbeing and behavioural presentations. We also measured if there had been an increase in the use of medication. Our focus was on the distress calls, which are requests for urgent clinical review to prevent a crisis.

Method. Six clinical areas across three Welsh health boards under the same specialist team were surveyed. Case notes and email correspondence were reviewed to obtain the number and content of crisis calls made to specialist CAMHS across an eight week period during the first UK COVID-19 lockdown. Data were gathered on frequency, purpose, and outcome of calls. Comparison data were also collected for the period October 2019 to March 2020.

Result. Pre-COVID-19: Two crisis calls were identified in two different areas during the pre-COVID period surveyed. Increases in medication and increases in respite care packages were made as a result.

During COVID-19 restrictions: 20 different initial distress calls made (children age 9 and 17 years old $(M=13.2) ; 75 \%$ were boys) across five of the six clinical areas. Of these 20 calls, 17 were active cases and 3 were new referrals. $95 \%$ of calls resulted in medication increases and there were few other interventions used due to COVID-19 constraints. Changes to the child's support system were discussed across all cases and return to school was highlighted as 